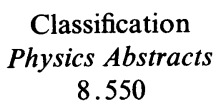

\title{
MISE EN ÉVIDENCE EXPÉRIMENTALE DES EFFETS DE FORME DANS L'ORTHOFERRITE DE TERBIUM
}

\author{
J. E. BOURÉE et J. HAMMANN \\ Service de Physique du Solide et de Résonance Magnétique, \\ Centre d'Etudes Nucléaires de Saclay, BP no 2, 91190 Gif-sur-Yvette, France
}

(Reçu le 11 octobre 1974, accepté le 6 janvier 1975)

\begin{abstract}
Résumé. - Les propriétés magnétiques de l'orthoferrite de terbium à basse température sont étudiées à partir de mesures d'aimantation réalisées entre $1 \mathrm{~K}$ et $10 \mathrm{~K}$ sur divers échantillons. Les courbes obtenues suivant les axes cristallographiques $x$ et $y$ permettent la détermination des paramètres physiques de l'ion $\mathrm{Tb}^{3+}$. Bien que les valeurs trouvées pour ces paramètres soient les mêmes, des mesures effectuées dans la direction $z$ font apparaître clairement deux types d'échantillons caractérisés par un comportement différent en fonction de la température. L'explication qui en est donnée, basée sur des différences de cinétique des domaines, utilise les prédictions relatives aux effets de forme dipolaires, qui sont contenues dans un diagramme de phase théorique publié antérieurement.
\end{abstract}

\begin{abstract}
The low temperature magnetic properties of terbium orthoferrite have been studied by means of magnetization measurements performed on various samples between $1 \mathrm{~K}$ and $10 \mathrm{~K}$. The curves obtained for the $x$ and $y$ crystallographic directions enable characteristic parameters of the $\mathrm{Tb}^{3+}$ ions to be determined. Although these values are the same, measurements along the $z$ axis clearly show up two kinds of samples with different behaviour as a function of temperature. This discrepancy is accounted for by shape effects which arise from the possibility of domain wall motion. These shape effects are due to dipolar interactions and are consistent with the predictions of the theoretical phase diagram published previously.
\end{abstract}

Introduction. - Dans une précédente publication [1] nous avons soulevé le problème théorique des effets de forme dus aux interactions dipolaires en nous basant sur le cas particulier de l'orthoferrite de terbium. Ce composé est connu surtout pour ses deux températures de réarrangement vers $3 \mathrm{~K}$ et $7 \mathrm{~K}[2,3,4]$, bien que certains échantillons ne semblent présenter qu'une seule transition [5,6]. Nous avons essayé d'analyser cette contradiction en montrant l'importance de l'énergie de champ démagnétisant dans la stabilité de la structure ferromagnétique, visible entre $3 \mathrm{~K}$ et $7 \mathrm{~K}$, par rapport à la structure antiferromagnétique, stable à $0 \mathrm{~K}$ dans tous les échantillons. Une première approche dans la référence [1] ne considère que les interactions dipolaires aussi bien à l'intérieur du système terbium qu'entre les systèmes fer et terbium. Une étude plus approfondie a été effectuée dans la même référence, en introduisant des termes d'échange et en utilisant des résultats expérimentaux obtenus sur des échantillons différents, qui permettent de préciser exactement les deux types de comportement.

Le but de cette publication est d'abord de présenter ces résultats expérimentaux, ensuite de discuter leur lien possible avec le diagramme de phase théorique [1] qui définit les domaines d'existence des différentes structures en fonction de la forme des échantillons.

1. Méthodes expérimentales. - 1.1 PrinciPe DeS MESURES. - Les mesures d'aimantation entre $1 \mathrm{~K}$ et $20 \mathrm{~K}$ présentées dans cet article sont obtenues par la méthode classique d'extraction : se plaçant à une valeur bien définie du champ magnétique, on extrait l'échantillon du centre d'une bobine captrice et on recueille un signal proportionnel à son aimantation aux bornes d'un fluxmètre. Le champ magnétique est fourni par des bobines supraconductrices dont l'homogénéité est de $10^{-3}$ dans un volume de $1 \mathrm{~cm}^{3}$. Une des bobines utilisées permet d'atteindre un champ maximum de $60 \mathrm{kOe}$.

Les températures sont obtenues dans un cryostat $\mathrm{He}^{4}$ (voir [7]) qui descend de $4,2 \mathrm{~K}$ à $1 \mathrm{~K}$ par pompage sur un bain d'hélium 4 liquide, la stabilisation de la température dans ce domaine étant d'environ $10^{-2} \mathrm{~K}$, et qui peut monter jusqu'à $30 \mathrm{~K}$ par chauffage et régulation de l'enceinte expérimentale. Les températures sont lues à l'aide d'une résistance au germanium (CGE, France) préalablement étalonnée, qui 
est mesurée par un pont de Wheatstone alternatif $(1 \mathrm{kHz})$ à détection synchrone, la précision relative obtenue étant de $10^{-4}$.

1.2 Echantillons. - Au cours de nos expériences, quatre types d'échantillons de $\mathrm{TbFeO}_{3}$ [8] ont été examinés : d'abord une poudre préparée par voie aqueuse, ensuite trois séries de monocristaux de provenance différente, obtenues par la méthode du flux [9]. Chacun des échantillons a été étudié aux rayons $X$ afin de vérifier la structure cristalline et la valeur des paramètres de maille. Aucune différence cristallographique n'a été observée et les valeurs des paramètres sont identiques à $10^{-3}$ près.

Des mesures spectrographiques effectuées sur les trois séries de monocristaux ont révélé un pourcentage d'impuretés, équivalent pour les trois cas, et qui se monte à environ $0,6 \%$ en poids de plomb provenant du fondant. Ces impuretés se manifestent soit comme inclusions à l'intérieur du volume de l'échantillon, soit comme substitution sur les sites de terbium (voir [9]) et sont certainement à l'origine des différences de comportement magnétique que nous allons discuter dans la suite.

2. Résultats expérimentaux. - Les mesures magnétiques réalisées sur les divers échantillons font apparaître deux types de comportement différent en fonction de la température :

a) Les poudres ainsi qu'une des séries de monocristaux (de provenance L.E.T.I. à Grenoble) présentent toujours, lorsqu'on abaisse la température, deux réorientations successives des moments du fer et du terbium, ce qui a déjà été observé par diffraction de neutrons $[2,3,4]$.

b) Les deux autres séries de monocristaux ne montrent qu'une transition à basse température, sans réorientation des moments du fer. Ce résultat a déjà été évoqué dans la littérature $[5,6]$.

Dans toute la suite, nous ne distinguerons donc que les échantillons notés I et II auxquels correspondent respectivement les deux types de comportement. Nous présentons d'abord les mesures permettant de définir les paramètres physiques, caractéristiques de l'ion $\mathrm{Tb}^{3+}$; puis nous mettons en évidence la structure magnétique stable à $0 \mathrm{~K}$, rencontrée dans tous les cristaux de $\mathrm{TbFeO}_{3}$. Enfin nous rendons compte des différences de comportement en fonction de la température, relatives aux échantillons I et II.

2.1 DÉTERMINATION DES PARAMÈTRES DE L'ION $\mathrm{Tb}^{3+}$. - De même que l'ion $\mathrm{Ho}^{3+}[10]$ dans $\mathrm{HoFeO}_{3}$, $\mathrm{Tb}^{3+}$ présente deux singulets de base bien séparés des niveaux supérieurs [11]. On peut ainsi caractériser ses propriétés magnétiques (voir $[1,10])$ par la séparation énergétique $\Delta$ entre les deux niveaux singulets, par le seul élément de matrice non nul $m_{\mathrm{s}}$ de l'opérateur moment magnétique entre ces deux états, enfin par les axes d'anisotropie liés à chaque position inéquivalente dans la maille; ces axes sont localisés dans le plan miroir $x O y$ et sont définis par la valeur de l'angle $\alpha$ avec l'axe $y$.

Les deux paramètres $m_{\mathrm{s}}$ et $\alpha$ peuvent être déduits des mesures d'aimantation suivant $x$ et $y$ à basse température $(\sim 1 \mathrm{~K})$ et en champs forts. Les courbes $m_{x}\left(H_{x}\right)$ et $m_{y}\left(H_{y}\right)$ correspondant à un échantillon I de forme sphérique sont données respectivement sur les figures 1 et 2 : elles présentent un comportement métamagnétique avec la particularité d'une double transition dans la direction $y$. Ces transitions seront étudiées en détail dans une publication ultérieure. Par extrapolation des parties en champ élevé, on trouve les valeurs à saturation

et

$$
m_{\mathrm{s}}^{x}=m_{\mathrm{s}} \sin \alpha=7,06 \mu_{\mathrm{B}}
$$

$$
m_{\mathrm{s}}^{y}=m_{\mathrm{s}} \cos \alpha=5,1 \mu_{\mathrm{B}},
$$

soit $m_{\mathrm{s}}=8,7 \mu_{\mathrm{B}}$ et $\alpha=54^{\circ}$. Ces résultats sont tout à fait compatibles avec $m_{0}=8,6 \mu_{\mathrm{B}}$ (moment magnétique spontané à $0 \mathrm{~K}[1,12])$ et $\alpha=50^{\circ}$ déduits des données de diffraction neutronique [2, 4], dans l'hypothèse où le mode $G$ du fer est dirigé suivant $x$.

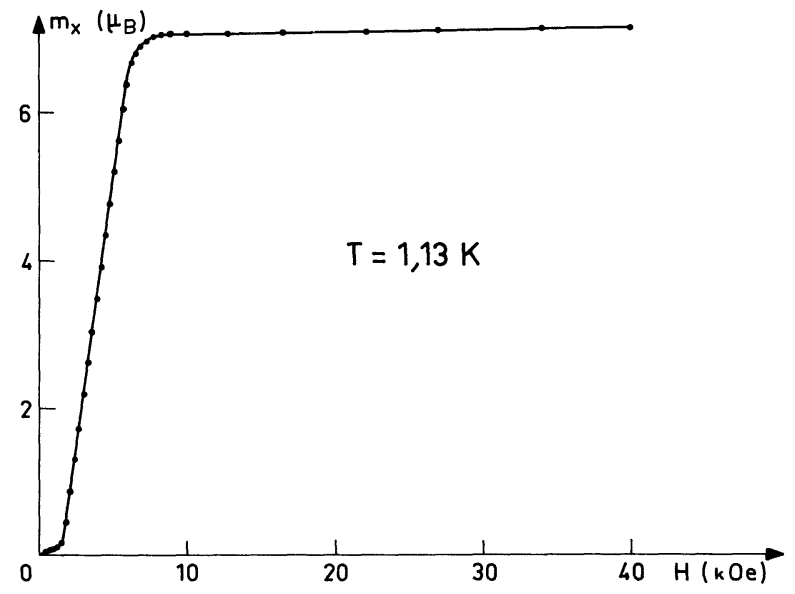

FIG. 1. - Aimantation d'un échantillon I en fonction du champ extérieur dans la direction $x$ à $1,13 \mathrm{~K}$.

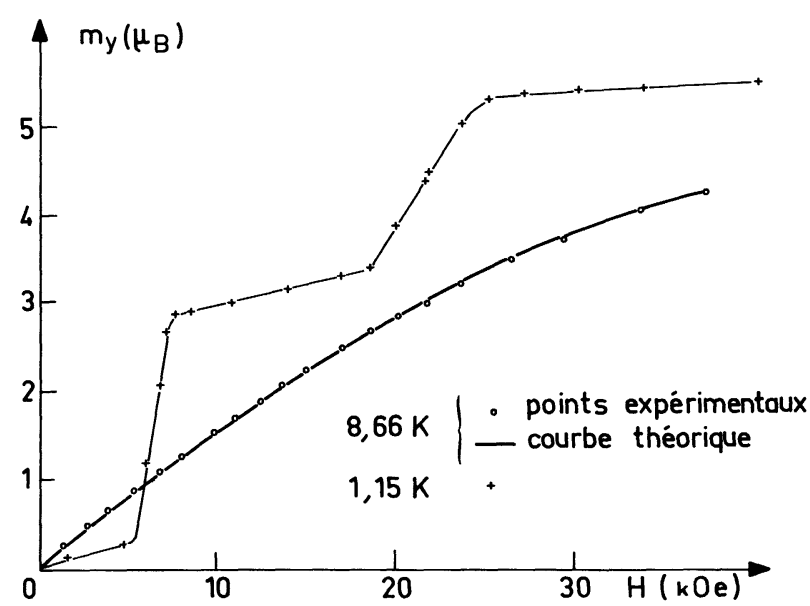

FIG. 2. - Courbes d'aimantation d'un échantillon I en fonction du champ extérieur dans la direction $y$ à $1,15 \mathrm{~K}$ et $8,66 \mathrm{~K}$. On a représenté en trait plein la courbe calculée à $8,66 \mathrm{~K}$ en donnant au paramètre $\Delta$ la valeur $\Delta / k_{\mathrm{B}}=1 \mathrm{~K}$. 
Le troisième paramètre $\Delta$ est déterminé à partir de la courbe $m_{y}\left(H_{y}\right)$ à $8,66 \mathrm{~K}$ (Fig. 2), c'est-à-dire dans une zone de température dans laquelle le système terbium est paramagnétique. La raison du choix de la direction $y$ vient du blocage des moments du fer dans le plan $x O z$ dû à la forte valeur du couplage Dzyaloshinski-Moriya des fer. En effet, par comparaison avec le cas de $\mathrm{YFeO}_{3}$, la composante du moment des fer suivant $y, \mu_{y}$, s'écrit pour un ion $\left(^{*}\right)$ :

$$
\mu_{y}=\frac{\mu}{1+\left[1+\left(\frac{H_{\mathrm{D}}}{H_{\mathrm{E}}}\right)^{2}\right]^{1 / 2}} \frac{H_{y}}{H_{\mathrm{E}}} .
$$

Prenant pour $\mu$ (moment à saturation des fer), $H_{\mathrm{D}}$ (champ d'échange Dzyaloshinski-Moriya) et $H_{\mathrm{E}}$ (champ d'échange isotrope), les valeurs adoptées dans [10], on trouve que $\mu_{y}$ atteint $1,5 \times 10^{-2} \mu_{\mathrm{B}}$ pour des champs de $40 \mathrm{kOe}$. Cette valeur est tout à fait négligeable devant celle des terbium et elle conduit surtout à une contribution négligeable des interactions $\mathrm{Fe}-\mathrm{Tb}$ à la valeur du champ local agissant sur un site de $\mathrm{Tb}$.

La composante de ce champ sur l'axe d'anisotropie du site considéré s'écrit (voir [10], table III) :

$$
\begin{aligned}
h=H_{y} \cos \alpha+\left(L_{3}+L_{4} \cos 2 \alpha+T_{1}\right) \frac{m_{y}}{\cos \alpha}+ \\
+\left[\frac{W}{\mu}+T_{2}\right] \mu_{y} \cos \alpha .
\end{aligned}
$$

Le premier terme correspond à la contribution du champ appliqué $H_{y}$, le second terme traduit l'effet des interactions $\mathrm{Tb}-\mathrm{Tb}$, isotropes, notées $L_{3}, L_{4}$ (voir [10]) et dipolaires notées $T_{1}$, le troisième terme rend compte des interactions $\mathrm{Fe}-\mathrm{Tb}$, isotropes, notées $W / \mu$ (voir [10]) et dipolaires notées $T_{2}$ $\left(W / \mu=-2,5 \times 10^{3} \mathrm{Oe} / \mu_{\mathrm{B}}[1], T_{2}=6,2 \times 10^{2} \mathrm{Oe} / \mu_{\mathrm{B}}\right) ;$ vu les ordres de grandeur de $\mu_{y}$, ce dernier terme disparaît devant la valeur du champ appliqué. Dans la zone paramagnétique, le second terme reste également petit devant le premier; on en a tenu compte comme correction, en adoptant les valeurs :

et

$$
L_{3}+L_{4} \cos 2 \alpha=-2,3 \times 10^{2} \mathrm{Oe} / \mu_{\mathrm{B}} \quad[1]
$$

$$
T_{1}=-2,14 \times 10^{2} \mathrm{Oe} / \mu_{\mathrm{B}} .
$$

La courbe expérimentale $m_{y}\left(H_{y}\right)$ à $8,66 \mathrm{~K}$ (Fig. 2) a donc été ajustée suivant la loi :

$$
\begin{aligned}
m_{y}=m_{\mathrm{s}} \cos \alpha \frac{a h}{\left(1+a^{2} h^{2}\right)^{1 / 2}} & \times \\
& \times \operatorname{Th} \frac{\Delta}{2 k_{\mathrm{B}} T}\left(1+a^{2} h^{2}\right)^{1 / 2}
\end{aligned}
$$

avec $a=2 m_{\mathrm{s}} / \Delta[12]$.

(*) BidauX, R., Bourée, J. E., Hammann, J., résultats non publiés.
On arrive ainsi au résultat $: \Delta / k_{\mathrm{B}}=1 \mathrm{~K}$. La précision n'est que de l'ordre de $0,5 \mathrm{~K}$, car la faible valeur de $\Delta$ réduit nettement son influence sur la forme de la courbe ajustée.

Le même type de mesures a été effectué avec un échantillon II, et les résultats sont équivalents mais avec une précision beaucoup plus faible à cause des difficultés d'évaluation du champ démagnétisant. En effet la petite taille des échantillons II disponibles ne nous a pas permis de les tailler en forme de sphère.

2.2 Structure magnétique Stable a $0 \mathrm{~K}$. Les mesures de diffraction neutronique effectuées à $1,5 \mathrm{~K}$ sur des poudres de $\mathrm{TbFeO}_{3}$ (échantillon $\mathrm{I}$ ) [2, $3,4]$ ont mis en évidence la configuration $A_{x} G_{y}$ des terbium, mais n'ont pas permis de faire la distinction entre les deux modes $G_{x}$ ou $G_{z}$ des fer. Nous avons montré dans un article théorique [1], que c'est le mode $G_{x} F_{z}$ qui devait être associé à la configuration $A_{x} G_{y}$ des terbium (structure antiferromagnétique VI, Fig. 5) : en effet, dans toute structure antiferromagnétique des terres rares, l'énergie d'interaction des deux systèmes est nulle par raison de symétrie, conduisant le système fer à garder la configuration $G_{x} F_{z}$ qu'il adopte à haute température sous l'effet de ses propres interactions : la preuve expérimentale la plus simple qui confirme ce résultat est donnée sur la figure 3 qui représente la courbe d'aimantation à $1,1 \mathrm{~K}$ dans la direction $z$ d'un échantillon I. Cette

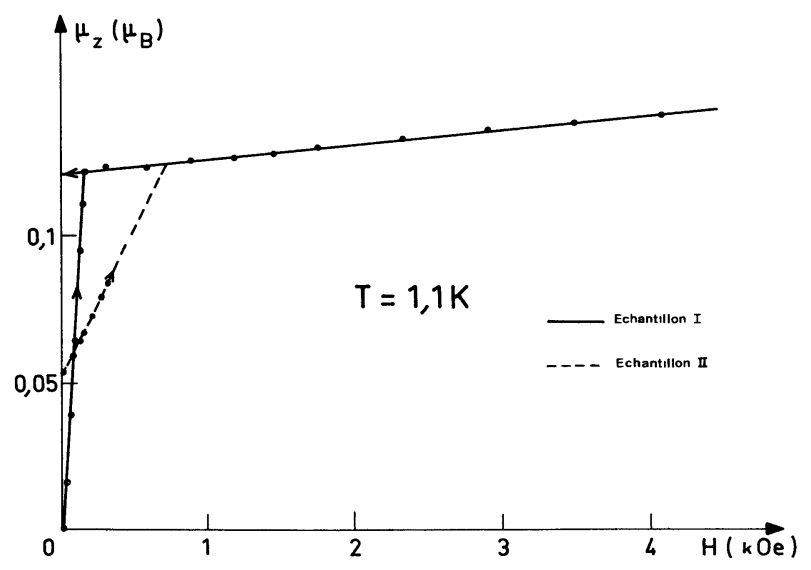

FIG. 3. - Aimantation des échantillons I et II en fonction du champ extérieur dans la direction $z$ à $1,1 \mathrm{~K}$. Les flèches pointant vers le haut ou le bas indiquent que les mesures correspondantes ont été faites en champ croissant ou décroissant.

courbe traduit le comportement ferromagnétique faible dans la direction $z$. On peut en déduire la valeur de l'aimantation spontanée résultante $\mu_{z}(0)=0,121 \mu_{\mathrm{B}}$; cette valeur est plus élevée que dans $\mathrm{HoFeO}_{3}$ ou $\mathrm{YFeO}_{3}$ [10] et est interprétée en affectant à l'interaction Dzyaloshinski-Moriya une valeur plus grande, soit $H_{\mathrm{D}}=2,1 \times 10^{5}$ Oe [1].

L'échantillon II donne le même résultat quantitatif pour l'aimantation spontanée à $1,1 \mathrm{~K}$ (Fig. 3), mais on observe d'importantes différences à champ faible, qui sont liées à des phénomènes d'hystérésis. Nous y reviendrons dans la discussion. 


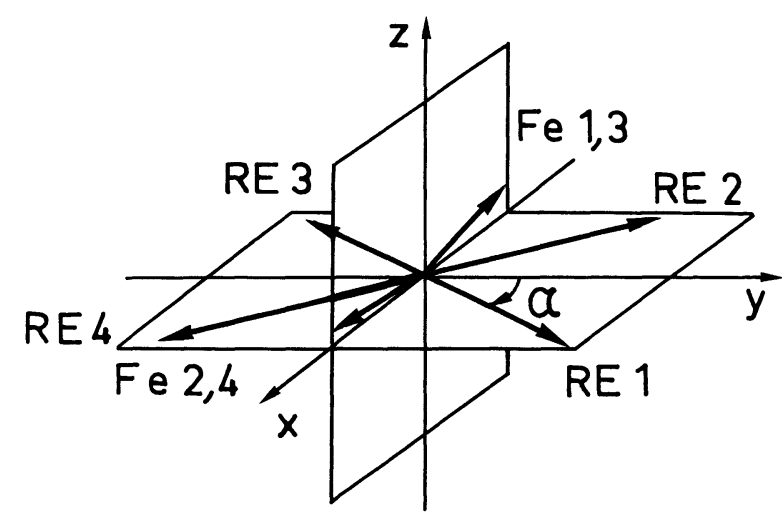

Fig. 4. - Configuration VI (structure antiferromagnétique $A_{x} G_{y}$ des moments de terre rare dans le plan $x O y$; voir [10], table III).

2.3 COMPORTEMENT EN FONCTION DE LA TEMPÉRATURE. - 2.3.1 Echantillon I. - Les expériences de diffraction neutronique [2, 4], de chaleur spécifique $[13,14,15]$ et les mesures d'effet Mössbauer [6] effectuées sur des poudres ont montré deux transitions magnétiques. Une première transition $\left(T_{\mathbf{R}_{1}} \sim 7 \mathrm{~K}\right)$ fait passer les moments du fer du mode $G_{x} F_{z}$ au mode $G_{z} F_{x}$ tandis que les moments du terbium, paramagnétiques au-dessus de $T_{\mathbf{R}_{1}}$, s'ordonnent suivant $F_{x} C_{y}$ (configuration I, Fig. 5). Une deuxième transition à $T_{\mathbf{R}_{2}} \sim 3,1 \mathrm{~K}$, caractérisée par une forte anomalie $\lambda$ de chaleur spécifique $[13,15]$, correspond à un réarrangement coopératif des moments du terbium suivant la configuration $A_{x} G_{y}$ et un retour des moments du fer suivant $G_{x} F_{z}$ (configuration VI, Fig. 4).

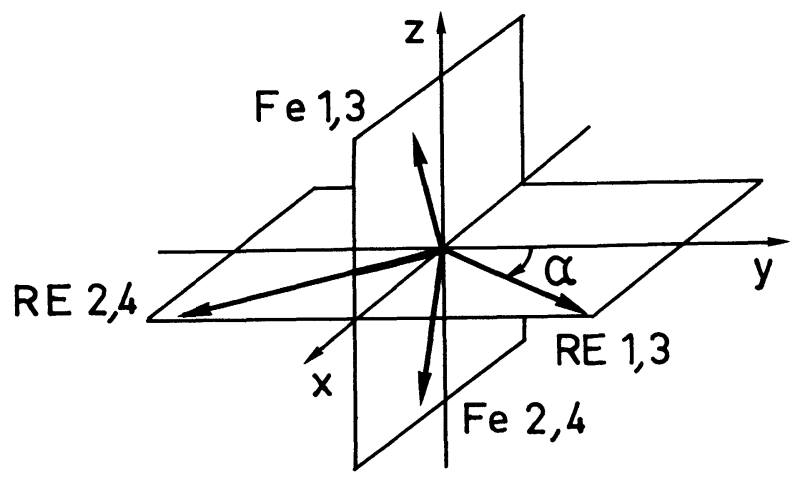

FIG. 5. - Configuration I (structure ferromagnétique $F_{x} C_{y}$ des moments de terre rare dans le plan $x O y$ ).

Ce comportement est bien illustré par la figure 6 qui représente un réseau de courbes d'aimantation réalisées suivant la direction $z$ en fonction de la température. La courbe correspondant au champ $H=180$ Oe montre parfaitement le domaine de température dans lequel la composante ferromagnétique des fer s'écarte de la direction $z$. Il est clair sur cette courbe que la rotation des fer se fait de façon progressive aussi bien du côté de $T_{\mathrm{R}_{1}}$ que du côté de $T_{\mathbf{R}_{2}}$, bien que des irrégularités de pente se mani-

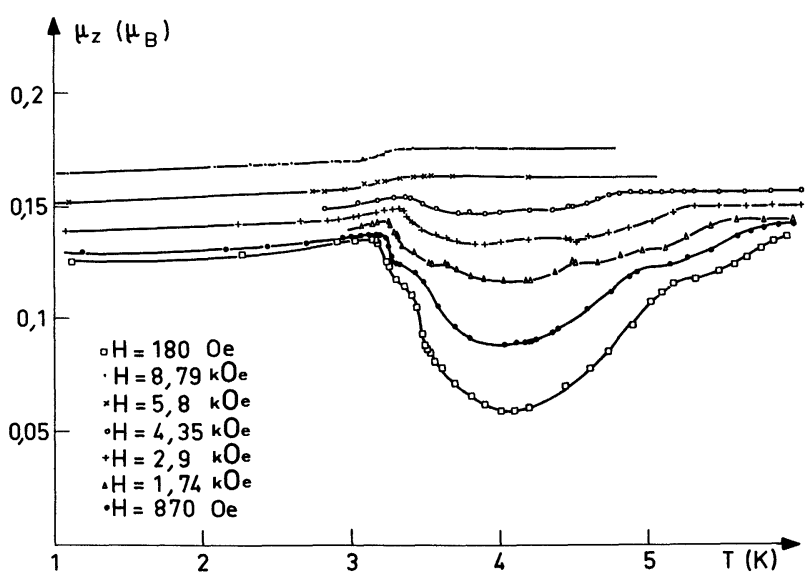

FIG. 6. - Réseau de courbes d'aimantation d'un échantillon I en fonction de la température pour différentes valeurs du champ appliqué dans la direction $z$.

festent dans chacune de ces zones de température. Le minimum d'aimantation observé, se situant vers $4 \mathrm{~K}$, n'est pas nul, peut-être parce que le retournement n'est pas complètement achevé, mais peut-être aussi parce qu'on voit une partie du fort moment ferromagnétique $F_{x}$ des terbium, à cause d'éventuels défauts d'orientation.

L'application d'un champ magnétique dans la direction $z$ a tendance à bloquer le moment ferromagnétique faible des fer dans cette direction. On voit ainsi sur la figure 6 , les courbes correspondant à des champs plus élevés, présenter des cuvettes de plus en plus étroites et de moins en moins profondes jusqu'à ce que, à partir de la courbe $H=5,8 \mathrm{kOe}$, il n'existe plus aucun retournement. Dans ce cas, les systèmes $\mathrm{Fe}$ et $\mathrm{Tb}$ restent toujours découplés, et il n'y a plus qu'une seule transition correspondant à l'établissement de l'ordre coopératif antiferromagnétique VI des moments du terbium sous l'effet de leurs propres interactions : cette transition $T_{\mathrm{N}}$ est indépendante de la valeur du champ appliqué suivant $z$ (dans la limite des champs dont nous disposons, laquelle reste loin de la valeur du champ d'anisotropie des terres rares \# $10^{5} \mathrm{Oe}$ ). A cause de la légère désorientation du cristal étudié, la transition $T_{\mathrm{N}}$ est visible sur les courbes

$$
H=5,8 \mathrm{kOe} \quad \text { et } H=8,79 \mathrm{kOe} \text { : }
$$

le décrochement existant vers $3,2 \mathrm{~K}$ correspond en effet à la diminution de la susceptibilité suivant $x$ du système des terres rares.

A partir du réseau de courbes de la figure 6, on peut ainsi tracer un diagramme de phase des échantillons I délimitant les domaines d'existence des structures paramagnétique, ferromagnétique (I) et antiferromagnétique (VI) en fonction de la température et du champ suivant $z$. Ce diagramme de phase est représenté sur la figure 7. A champ nul, il donne les deux valeurs : $T_{\mathrm{R}_{1}} \sim 6,2 \mathrm{~K}$ et $T_{\mathbf{R}_{2}} \sim 3,15 \mathrm{~K}$; $T_{\mathrm{N}}$ est obtenu par extrapolation à champ nul de la 
ligne de transition PARA-AF qui n'est pas exactement verticale à cause de la désorientation. On trouve $T_{\mathrm{N}} \sim 3,45 \mathrm{~K}$.

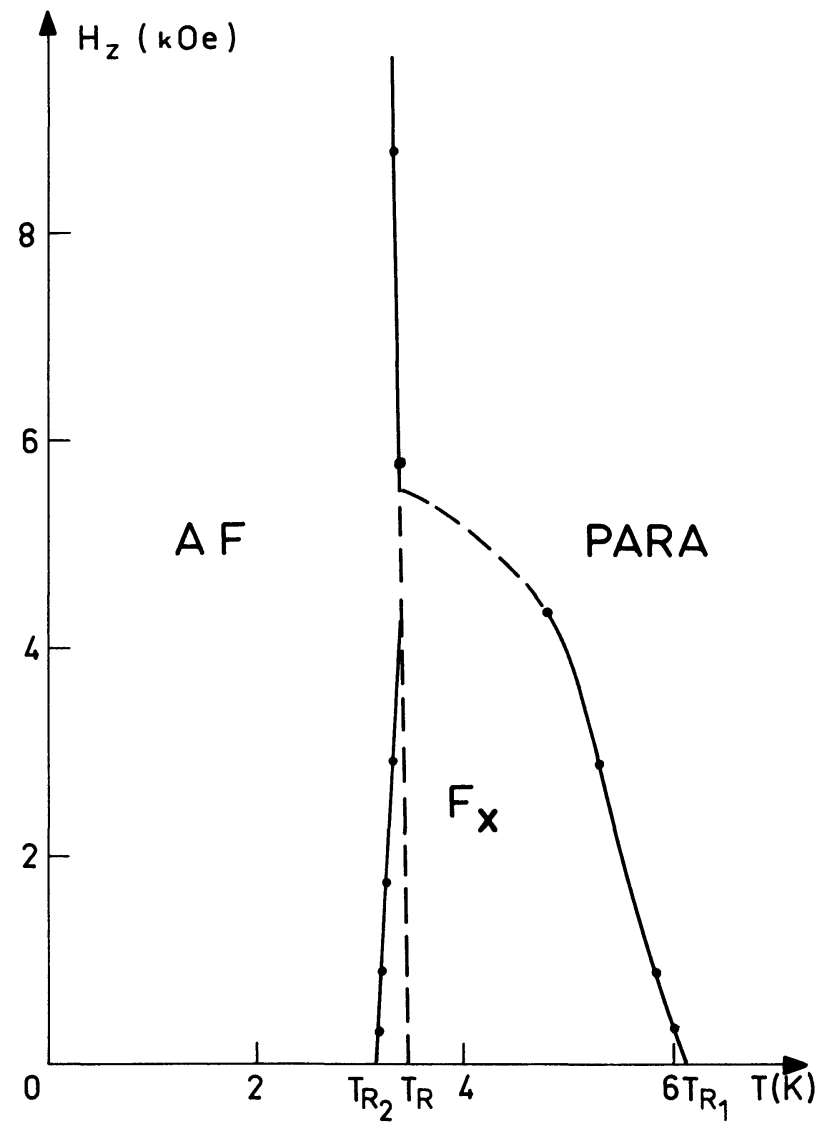

Fig. 7. - Diagramme de phase expérimental d'un échantillon I de $\mathrm{TbFeO}_{3}$ dans la direction $z$ en fonction de la température. PARA, $F_{x}$ et AF se réfèrent respectivement aux phases paramagnétique, ferromagnétique (configuration I) et antiferromagnétique (configuration VI) du système terre rare.

2.3.2 Echantillon II. - Comme nous l'avons mentionné plus haut, Belov et al. [5] effectuant des mesures de couple magnétique n'ont remarqué sur les échantillons qu'ils utilisaient qu'une transition de type paramagnétique-antiferromagnétique. Plus récemment Sivardière et al. [6] ont remarqué par diffraction de neutrons que certains monocristaux de $\mathrm{TbFeO}_{3}$ ne présentaient pas de réorientation des fer de $G_{x}$ vers $G_{z}$.

Nous avons effectué des mesures d'aimantation suivant $z$ en fonction de la température, sur des échantillons de type II, dans des champs aussi faibles que possible. On remarque sur la figure 8 que le comportement de l'aimantation à champ faible est équivalent à celui qui a été observé sur les échantillons I en champ fort (voir Fig. 6). Il n'existe pas de retournement du faible moment ferromagnétique des fer, et comme sur les courbes de la figure 6 , on peut localiser la transition $T_{\mathrm{N}}$ correspondant à l'ordre antiferromagnétique des moments de terbium sous l'effet de leurs propres interactions, grâce à une légère désorientation du cristal. On voit que $T_{\mathrm{N}}$ est encore de l'ordre de 3,4 K.

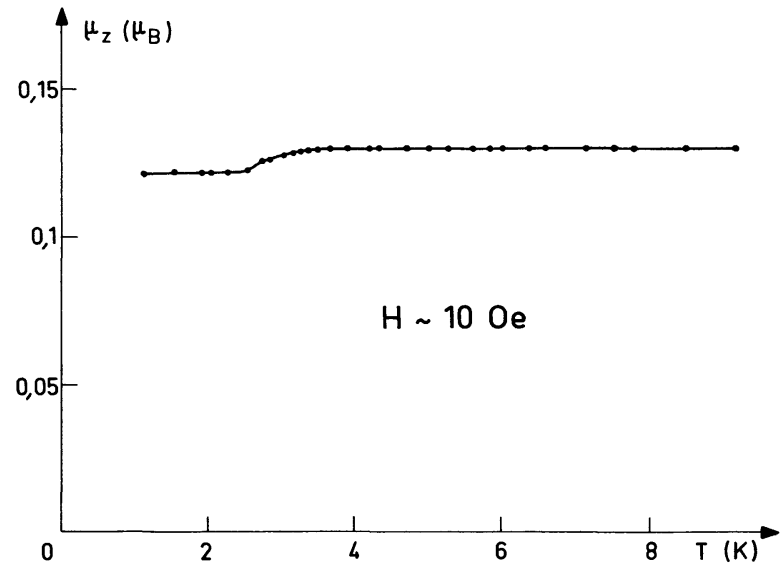

FIG. 8. - Aimantation suivant $z$ d'un échantillon II en fonction de la température, dans un champ très faible $(\sim 10 \mathrm{Oe})$.

Pour essayer de confirmer cette valeur de $T_{\mathrm{N}}$, des mesures d'aimantation suivant la direction $x$ en fonction de la température, et pour un champ appliqué d'environ 300 Oe, ont été reportées sur la figure 9. Le comportement antiferromagnétique est confirmé. La courbe présente un point d'inflexion vers $3,45 \mathrm{~K}$, valeur qui peut être considérée comme étant la température de transition; le très large maximum obtenu au-dessus de cette température est dû à une légère rotation du système fer qui induit un couplage $\mathrm{Fe}-\mathrm{Tb}$, ce qui augmente la valeur du champ local en un site de $\mathrm{Tb}$. A cause de cet effet, il est difficile de déterminer $T_{\mathrm{N}}$ avec une bonne précision en utilisant uniquement des mesures magnétiques, mais les ordres de grandeur qui viennent d'être donnés suffisent pour comprendre les différents comportements observés.

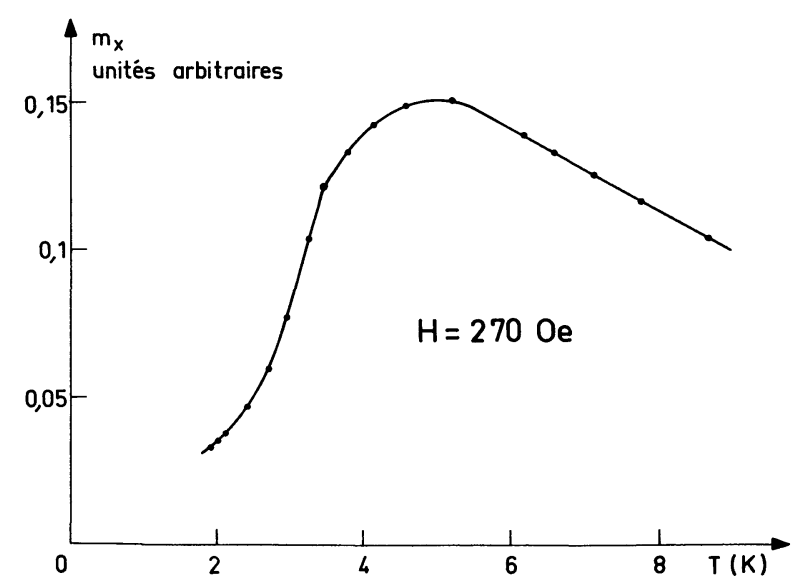

FIG. 9. - Aimantation suivant $x$ d'un échantillon II en fonction de la température, dans un champ de 270 Oe.

Discussion. - L'identité de comportement dans la phase paramagnétique, l'équivalence de la structure à $0 \mathrm{~K}$, ainsi que l'égalité des températures $T_{\mathrm{N}}$ entre les échantillons I et II, permettent de conclure à l'invariance des paramètres physiques responsables des propriétés magnétiques de $\mathrm{TbFeO}_{3}$, en dehors d'une peu plausible différence entre les interactions 
$\mathrm{Fe}-\mathrm{Tb}$. Comment expliquer alors la présence d'une zone intermédiaire ferromagnétique dans un type d'échantillons et non dans l'autre?

La réponse la plus satisfaisante à cette question a été obtenue en analysant les phénomènes d'hystérésis observés au cours des mesures d'aimantation dans la direction $z$. Lorsqu'on coupe le champ magnétique agissant sur un échantillon I, après l'avoir saturé à basse température, dans la phase $F_{z}$ des fer, on observe une décroissance lente de l'aimantation spontanée jusqu'à son annulation totale après environ $20 \mathrm{~min}$; ce résultat est reporté sur la figure 10 .

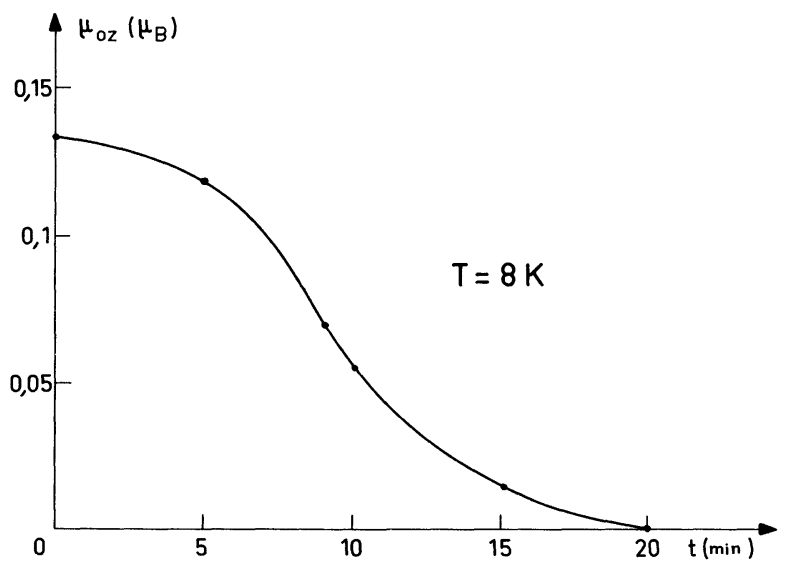

FIG. 10. - Variation de l'aimantation spontanée suivant $z$ d'un échantillon I en fonction du temps à $8 \mathrm{~K}$.

La figure 11 représente le cas d'un échantillon de type II : l'échelle de temps dans ce cas est fondamentalement différente. La courbe de décroissance a une pente, très faible au début, qui s'annule complètement lorsque l'aimantation spontanée a atteint environ la moitié de sa valeur à saturation. La figure 3 représente une courbe de seconde aimantation à partir de cette valeur limite. On voit l'importante hystérésis qui la caractérise. Pour arriver à annuler complètement l'aimantation spontanée à champ nul, il faut réchauffer le cristal au moins jusqu'aux températures de l'azote liquide.

La distinction qui est ainsi mise en évidence entre les deux types d'échantillons, signifie que dans le cas d'une structure ferromagnétique, les échantillons I parviennent à une meilleure minimisation de l'énergie de champ démagnétisant par formation de domaines d'aimantation macroscopique opposée. Cette minimisation est complète si chacun de ces domaines a une forme telle que son facteur de champ démagnétisant $\mathcal{N}$ dans la direction du moment résultant soit nul. Les échantillons II, par contre, doivent toujours présenter,

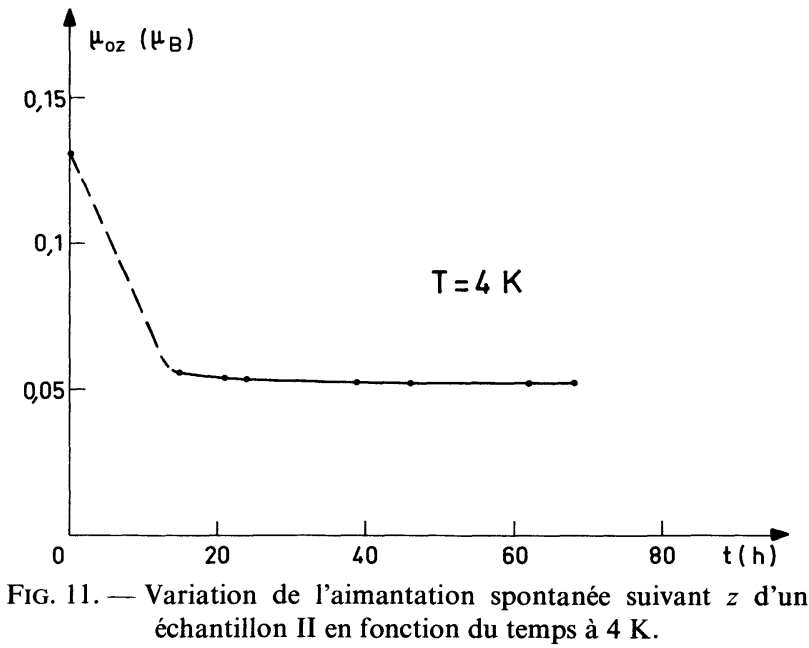

dans le cas d'une transition ferromagnétique à basse température, un moment spontané macroscopique non nul, et sont ainsi soumis à un champ démagnétisant fonction de la forme extérieure de l'échantillon.

$\mathrm{Si}$ on se reporte alors au diagramme de phase théorique qui est publié dans la référence [1] et qui met en évidence les effets de forme issus des interactions dipolaires, la comparaison entre $\mathcal{N}=0$ et $\frac{\mathcal{N}}{4 \pi}>0,13$ permet de rendre compte de la différence de comportement magnétique entre les échantillons I et II.

On peut enfin essayer de donner une explication aux différences de cinétique des domaines en invoquant des différences de répartition des impuretés de plomb à l'intérieur des cristaux. En effet des mesures de spectrographie ont montré que dans les monocristaux de type I, les impuretés étaient concentrées dans certaines régions du cristal, alors qu'elles se trouvent uniformément réparties dans les échantillons II, ceci pour une concentration globale équivalente $(0,6 \%$ en poids) pour les deux types. Il en résulte que pour les types I, la distance moyenne entre impuretés est très supérieure à la dimension des domaines, alors qu'elle peut être du même ordre de grandeur pour les types II avec pour conséquence un blocage des parois à basse température. Cette explication est compatible avec le fait que les échantillons en poudre, fabriqués par voie aqueuse et ne contenant donc pas de plomb, sont tous de type I.

Remerciements. - Les auteurs tiennent à remercier ici M. R. Gérard-Deneuville pour sa contribution active à bon nombre de manipulations, ainsi que M. R. Bidaux pour les discussions fructueuses qu'ils ont eues avec lui tout au long de ce travail.

\section{Bibliographie}

[1] Bidaux, R., Bourée, J. E., Hammann, J., J. Phys. Chem. Solids II.

[2] Bertaut, E. F., Chappert, J., Mareschal, J., Reboulllat, J. P. and Sivardière, J., Solid State Commun. 5 (1967) 293.
[3] Mareschal, J., Sivardière, J., De Vries, G. F. and Bertaut, E. F., J. Appl. Phys. 39 (1968) 1364.

[4] Mareschal, J. and Sivardière, J., J. Physique 30 (1969) 967. 
[5] Belov, K. P., Kadomtseva, A. M., Ledneva, T. M., Ovchinnikova, T. L., Ponomarev, Ya. G. and Timofeeva, V. A., Sov. Phys. Solid State 9 (1968) 2193.

[6] Belakhovsky, M., ChapPert, J., Rouskov, T., Sivardière, J., J. Physique Colloq. 32 (1971) C 1-492.

[7] Testard, O., Thèse d'Université, Orsay (1966), Rapport CEA-R-3073.

[8] $\mathbf{M}^{\mathrm{me}}$ CARCAILleT, C. (CEN-Saclay) nous a fourni les échantillons en poudre. La première série de monocristaux a été préparée par GuINET, P. (L.E.T.I. Grenoble), la deuxième par LEGRAND, S. (CEN-Saclay), enfin la troisième série nous a été gracieusement fournie par le Prof. White, R. L. de l'Université de Stanford.
[9] Remeika, J. P. and Kometani, T. Y., Mat. Res. Bull. 3 (1968) 895.

[10] Bidaux, R., Bourée, J. E., Hammann, J., J. Phys. \& Chem. Solids 35 (1974) 1645.

[11] Rossat-Mignod, J. and Tcheou, F., J. Physique 33 (1972) 423.

[12] Bidaux, R., Gavignet-Tillard, A., Hammann, J., J. Physique 34 (1973) 19.

[13] De Combarieu, A., Michel, J. C., Peyrard, J. et Sivardière, J., Int. Conf. Low Temp. Physics, Saint-Andrews, 1968.

[14] De Combarieu, A., Mareschal, J., Michel, J. C. et SivarDIÈRE, J., Solid State Commun. 6 (1968) 257

[15] Peyrard, J., Sivardière, J., Solid State Commun. 7 (1969) 605. 